\title{
多尺度共轭微孔聚合物的可控合成
}

\author{
吴可义 郭佳* \\ (聚合物分子工程国家重点实验室 高分子及其先进复合材料协同创新中心 复旦大学高分子科学系 上海 200433)
}

\begin{abstract}
摘要 自 2007 年首次报道以来, 作为一种由共轭单元构建的三维聚合物网络骨架, 共轭微孔聚合物(Conjugated Microporous Polymer, CMP)通常都是以不溶不熔的固体粉末形式存在; 尽管这种材料结合了优异的多孔性、稳定的骨架结 构以及多样化的功能, 显示了在众多领域的应用价值和广阔前景, 但又始终面临着自身性质带来的难以解决的加工性 问题. 为了让这种材料充分发挥自身优点, 应用于除吸附分离等以外的光电、传感、催化等能源环境相关的领域, 需要 在多尺度范围内调控 CMP 生长和形成, 获得微纳尺度的 CMP 微球以及宏观尺度的 CMP 薄膜、涂层或是凝胶, 从而提 高其溶液性质以便于进一步加工处理, 或是直接获得可用于构筑器件的薄膜. 从目前的研究进展来看, 一共有四种研 究策略来解决这一问题, 分别是设计合成: (1)可溶性 CMP 聚合物, (2)溶液可分散 CMP 纳米微球, (3) CMP(复合)薄膜, (4)有机相 CMP 化学凝胶. 这些工作采用了新的聚合方法、催化剂或功能单体, 使 CMP 材料初步实现了溶液中的加工、 组装、复合以及器件的构筑，展示了在光学传感、光电转换、能量存储、非均相催化等优异的性质. 尽管目前已报道 的工作仍旧面临较多的局限性, 然而基于创新的思路和大量的探索, 这类新型的功能高分子材料将会逐步成为一个重 要的多孔材料分支, 具有光明的发展前景.
\end{abstract}

关键词＼cjkstart共轭高分子; 多孔性; 加工; 可溶性; 微球; 薄膜; 凝胶

\section{Controllable Synthesis of Multi-Scale Conjugated Microporous Polymer}

\author{
$\mathrm{Wu}$, Keyi Guo, Jia*
}

(State Key Laboratory of Molecular Engineering of Polymers, Collaborative Innovation Center of Polymers and Polymer Composite Materials, Department of Macromolecular Science, Fudan University, Shanghai 200433)

\begin{abstract}
As it was firstly reported in 2007, conjugated microporous polymer (CMP) has been constructed by a diversity of conjugation building blocks towards a three-dimensional rigid organic framework with the form of insoluble and infusible solid powders. Although CMP has showed the collective characteristics such as exceptional porosity, stable network structure and versatile functionality for potential applications and broad prospects in many fields, the problem of processability concerning this kind of material has not been overcome yet. To take full advantage of their features and break through the application scopes from adsorption and separation to energy and environment such as photoelectric transformation, sensing and catalysis, modulation of growth and formation of CMP in multiple scales is highly anticipated, giving rise to the micro/nanometer-size CMP microspheres and macroscopic CMP films, coatings or gels. Unambiguously, such well-organized forms either have the improved solution properties for further processing, or appear membranes directly assembled into devices. Looking back at the progress of CMP studies in recent years, there are four strategies reported to explore multi-scale CMPs, including (1) soluble CMP-like polymer, (2) solution-dispersible CMP microspheres, (3) CMP-based (composite) film, and (4) CMP-supporting organogel. In these studies, the novel polymerization methods, new catalysts or functional monomers were adopted; the resulting CMPs could be processed, assembled or combined with other materials in solution, and have greatly promoted the performances of optical sensing, photoelectric conversion, energy storage and heterogeneous catalysis on intended devices. It is noted that the reported methodologies have some limitation, but upon the creative ideas and vast explorations, CMP is going to be one important branch of porous materials with promising perspectives.
\end{abstract}

Keywords conjugated polymer; porosity; processability; solubility; microsphere; film; gel

\section{1 引言}

回顾百年来材料的发展历史, 在二十世纪数十年中 多孔材料始终吸引着广泛而浓厚的研究兴趣, 特别是丰 富多样的无机多孔材料始终引领并代表着新材料发展
的广阔前景和快速趋势. 近二十年来, 有机/无机杂化以 及有机多孔材料相关领域同样展现了旺盛的研究活力, 涌现出了大量的研究成果, 其中最具代表性的材料分别 是金属有机骨架(Metal Organic Framework, MOF) ${ }^{[1]}$ 和多 孔有机聚合物(Porous Organic Polymer, POP) ${ }^{[2]}$. 与以往

*E-mail: guojia@fudan.edu.cn

Received February 21, 2015; published May 6, 2015.

Project supported by the National Natural Science Foundation of China (No. 21474015) and the Science and Technology Commission of Shanghai Municipality (No. 14ZR1402300).

项目受国家自然科学基金(No. 21474015)与上海市科委(No. 14ZR1402300)资助. 
不同的是, 设计这些材料需要从分子角度出发, 从而构 筑结构组成更加多样化的多孔骨架. 以多孔有机聚合物 为例，设计构筑分子的空间构型和功能侧基、成键方式、 反应类型等, 就能够得到各种不同孔径尺寸、理化性质 的多孔有机聚合物. 目前国际上最为知名的多孔有机聚 合物主要包括以下四类: (一)共价有机骨架(Covalent Organic Framework, $\mathrm{COF})^{[3]}$; (二) 超交联聚合物 (Hyper-Crosslinking Polymer, HCP) ${ }^{[4]}$; (三)内在多孔性聚 合物(Polymer of Intrinsic Microporosity, PIM) ${ }^{[5]}$; (四)共 轭微孔聚合物 (Conjugated Microporous Polymer, $\mathrm{CMP})^{[6]}$. 本文将基于最后一类共轭微孔聚合物的进展 作细致阐述.

共轭微孔聚合物是一类由全共轭分子链围筑的、具 有三维网络结构的、微孔型有机高分子材料. CMP 在微 观结构上由于共轭单元的刚性以及共轭成键的方式, 因 此在溶剂分子抽提后, 骨架结构能有效的支撑起微孔通 道, 而不像共轭小分子或是线性链那样通过 $\pi-\pi$ 作用堆 叠而形成致密无孔的聚集体. 如此, 该材料既具有某些 共轭高分子的光电性质, 又能够提供稳定的内在多孔 性, 显示出了非常独特、优异、可设计的使用性能.

自 2007 年利物浦大学的 Cooper 教授课题组 ${ }^{[6 a]}$ 合成 出首例 CMP 以来, 围绕这类材料而进行的研究集中在: (1)使用不同类型的反应及反应单体, 如 Yamamoto 反 应、Suzuki 反应、Sonogashira 反应等来偶联功能单体; (2) 使用不同对称性的共轭单体, 如 $\mathrm{C}_{2}, \mathrm{C}_{3}, \mathrm{C}_{4}$ 和 $\mathrm{C}_{6}$ 等拓扑 形态来构建不同孔性质的骨架; (3)采用不同功能的共轭 单体聚合或者功能化修饰, 从而展现了 CMP 在气体吸 附与分离 ${ }^{[6,7]}$ 、异相催化 ${ }^{[8]}$ 、光能捕获及转移 ${ }^{[9]}$ 、超级电 容器 ${ }^{[10]}$ 、化学传感 ${ }^{[11]}$ 等诸多领域的重要使用价值. 但是, 就实际应用而言, CMP 材料由于多官能团间的交联聚合 得到三维网络骨架, 必然呈现不溶不熔的性质, 无法通 过传统方法直接形成涂层、薄膜或是形态固定的模块. 因此, 较难用于器件加工、材料复合、功能掺杂等进一 步的后续处理. 然而, 回顾其他材料的发展历程会发现, 无论是从远古的青铜材料以及之后的钢铁材料, 抑或是 现代的聚合物材料, 都是在掌握其熔化加工性质后, 才 将之应用到民用或军事的方方面面. 无法人工实现溶熔 加工的岩石材料, 只能采用打磨等方式当作建材使用, 而可以溶熔的聚合物材料甚至可以通过 3D 打印这样的 新技术制备复杂结构形态的成品. 尽管在提高 CMP 的 溶液分散性、成膜性以及微纳尺度的可控合成等方面获 得了一系列成果, 然而在此方面的探索仍在进行, 取得 某些实际应用中的突破将一直是 CMP 材料发展的难点 和热点. 近年来的一些综述文章也将突破加工性与材料 完整性作为这类材料发展需要面对的“极其重要的挑 战, 控合成进行总结和展望.

\section{2 多尺度 CMP 材料的可控合成思路}

\section{1 可溶性 CMP 聚合物的合成}

在 2012 年, 首次报道共轭微孔聚合物的 Cooper 课 题组，以四溴取代的萠单体作为交联骨架，与二溴取代 的萠单体共聚, 通过调控分子量以及引入末端的叔丁 基，实现共轭微孔聚合物的溶解性(图 1$)^{[6 \mathrm{~m}]}$.

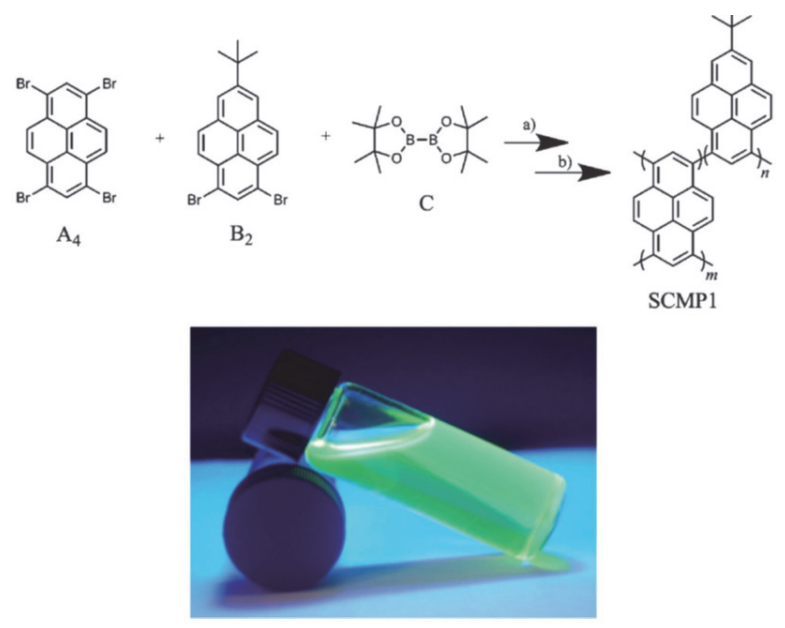

图 1 两步一锅法合成可溶性共轭微孔聚合物(改编自文献 $[6 \mathrm{~m}]$ )

Figure 1 Two-step, one-pot synthesis of a soluble conjugated microporous polymer. (Adapted from the literature [6m])

这种类似树枝状的 CMP 可以在二氯甲烷、甲苯、 四氢呋喃等常见有机溶剂中溶解，通过旋涂可以制备均 匀的薄膜材料, 很好解决了 CMP 材料的加工性问题. 然而从分子水平上来看, 这种方法得到的 CMP 聚合度 较低, 其重复单元数大约在 20 个, 其聚合物结构更象是 交联的树枝状大分子; 并且只有在不良溶剂中快速沉 淀, 才能保证一定的多孔性, 如果通过溶剂缓慢挥发形 成薄膜后，其比表面积仅有 $12 \mathrm{~m}^{2} / \mathrm{g}$, 多孔性受后处理的 条件影响很大.

通过这一方法得到的 CMP 分子量大约为 5000, 并 且反应中涉及的聚合程度的控制较难把握, 因此很少有 报道将此方法应用于其他类型可溶性 CMP 的制备, 但 这一思路非常有借鉴意义. 在一些传统的体型高分子聚 合方法中, 为了适应加工要求, 常常先制备线性低聚物 链, 然后在模具中交联得到三维骨架的高聚物. 这种两 步法也可应用于 CMP 的合成. 在 2014 年, Cooper 课题 组 ${ }^{[13]}$ 在前期工作的基础上, 进行了可溶性 CMP 的系统 研究工作. 制备了 18 种具有内在多孔性的线性聚合物 PIM, 并调节 4 种聚合物的分子量, 可以得到多孔性随 之变化的 PIM 薄膜. 在此基础上, 对于其中侧基含有烯 基的 PIM 体系，成功通过双键交联得到了化学稳定的多 孔薄膜. 同样在 2014 年, Yavuz 课题组 ${ }^{[14]}$ 也采用这一策 略, 以硅烷作为聚合反应前驱体, 溶解在极性溶剂中并 挥发成膜, 然后在高温下使硅烷交联, 形成稳定多孔的 
共轭薄膜体系. 这种方法适用于以咪唑、二唑、三唑、 四嗪为成键产物的交联方式, 在 CMP 材料的合成中确 实具有相当的实用价值.

\section{2 溶液可分散的微纳尺度 CMP 设计合成}

在溶液中, 绝大部分 CMP 颗粒无法长期稳定分散, 虽然有报道称某些组成的 CMP 可以部分溶胀, 然而由 于聚合物的尺度和表面性质缺乏调控, 因此常以聚集体 形式悬浮在液相中. 最早尝试解决 CMP 加工问题的思 路就是考虑提高 CMP 的溶液性质, 而有效调控 CMP 的 微观尺度、形态和表面性质, 提高其在溶液中的分散稳 定性, 从而加工成涂层或薄膜, 将是一条行之有效的策 略. 在此思路的指导下, 我们课题组近年来尝试用乳液 聚合体系开发了一系列的微纳尺度 CMP 微球, 研究了 CMP 在受限空间内的可控生长、表面修饰、功能复合、 薄膜制备以及多样化的应用.

2011 年, 我们课题组在传统的细乳液聚合方法上 进行了大量尝试, 用甲苯作为油相溶解聚合单体和催化 剂, 通过均质器配成水包油的细乳液反应体系. 在适合 温度下于油相液滴中催化聚合, 最终形成乳化剂稳定分 散的 CMP 微球(图 2) ${ }^{[8 \mathrm{e}]}$. 通过 click 反应, 在表面修饰功 能基团, 获得在不同极性溶剂中稳定的胶体溶液, 并展 现了优异的苂光性质. 由于高比表面积以及刚性骨架支 撑的稳定孔道, 我们在微球中负载了钯纳米粒子, 用于 Suzuki 反应的非均相催化. 其催化效果 TOF 高达 44100 $\mathrm{h}^{-1}$, 在多次反复使用中也展现了优异的转化率和极低 的钯流失率; 催化速率是市场销售的钯碳催化剂的三 倍, 接近完全转化, 使得最终产物的提纯分离得以简化, 是目前报道的、适用于该反应的、最好的非均相催化剂 之一.

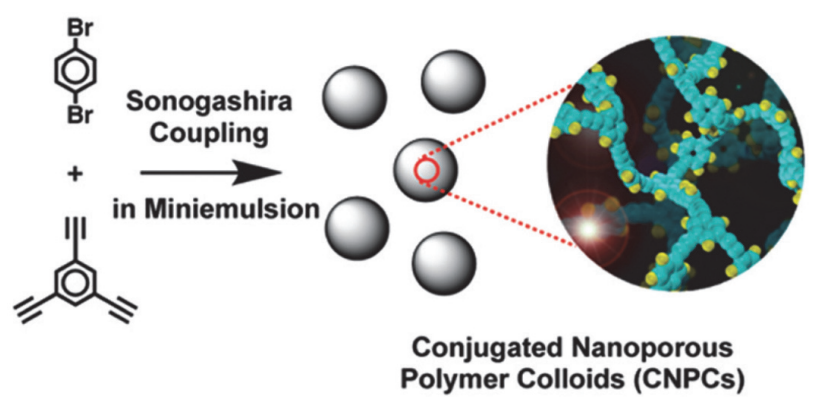

图 2 在水包甲苯细乳液体系中合成 CMP 纳米粒子. (改编自文献 [8e])

Figure 2 Schematic illustration of CMP microspheres synthesized by the Sonogashira coupling reaction in a toluene-in-water miniemulsion. (Adapted from the literature [8e])

2012 年, 在此基础上, 我们仍基于水包油的细乳液 反应体系, 引入表面修饰有苯溴结构的磁性 $\mathrm{Fe}_{3} \mathrm{O}_{4}$ 纳米 粒子, 使其作为含卤单体的一部分参与端炔与溴基团的 Sonogashira 偶联反应, 最终得到既具有超顺磁性, 又具 有 CMP 的发光性和多孔性的、有机/无机杂化的共轭微
孔聚合物微球 ${ }^{[11 c]}$. 由于 $\mathrm{Fe}_{3} \mathrm{O}_{4}$ 通过共价键参与 $\mathrm{CMP}$ 骨 架的构筑，其结构分为有机高分子构筑的微孔区以及无 机纳米粒子参与构筑的介孔区. 我们基于微球整合了多 孔性、苂光性、磁性和催化性能的特点, 通过多功能的 协同作用，实现了在液相中高速、灵敏、特异的光学检 测硝基苯酚类化合物.

端炔基和卤素的 Sonogashira 偶联反应在较高的反 应温度和较长的反应时间下，可以提高聚合程度，然而 这将会影响细乳液体系的稳定性; 倘若降低反应温度， 会导致反应程度不够，多孔性下降等问题. 2013 年，我 们采用了室温下端炔基氧化均聚的方法，将四炔基四苯 基锌卟啉在细乳液体系中聚合成 CMP 微球 ${ }^{[11 \mathrm{~d}]}$. 由于该 反应在室温下进行, 也不需要隔绝氧气操作, 因此更加 适合反应条件温和的乳液体系，同时也不会损失多孔性 质. 所得到的 CMP 微球由于富含四苯基锌卟啉活性中 心, 在锌卟啉配体与 $\mathrm{SO}_{2}$ 交换中, 微球的颜色会发生变 化, 因此可以实现 $\mathrm{CMP}$ 微球的分散液对于 $\mathrm{SO}_{2}$ 气体的 变色检测，以及更简便的试纸检测，其适用性及灵敏性 都非常优秀(图 3), 充分体现了可分散性纳米 CMP 微球 的应用优势.

a)

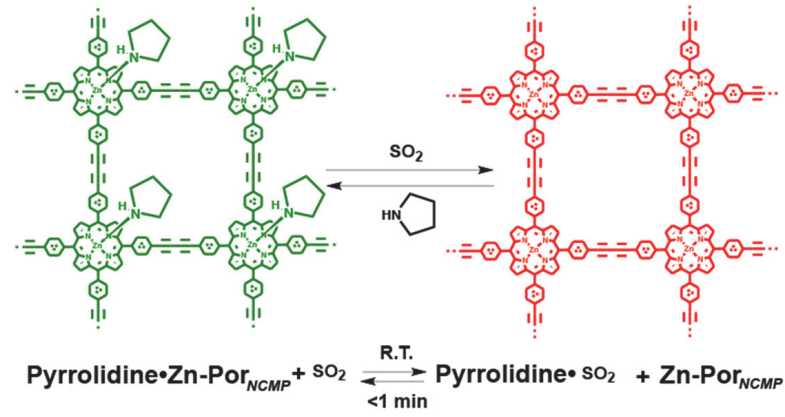

b)

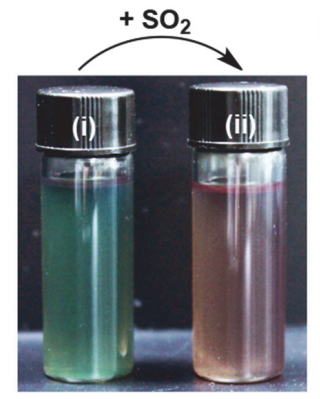

c)

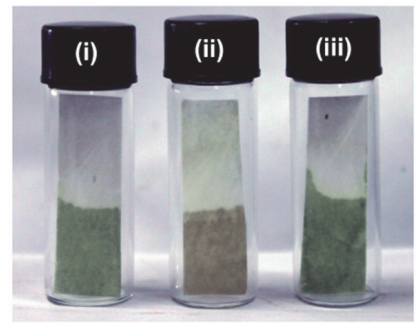

图 3 溶液分散的锌卟啉 CMP 微球变色检测二氧化硫气体: (a)机理, (b)液相检测, (c)试纸检测(改编自文献[11d])

Figure 3 (a) Mechanism of colorimetric detection of $\mathrm{SO}_{2}$ gas using porphyrin-Zn-based CMP microspheres, and the photographs to show the color changing in solution (b) and testing papers (c). (Adapted from the literature [11d])

在 2014 年, 我们进一步研究了乳液体系中的 Sonogashira 偶联反应, 采用两步法合成多孔性极大提 高的 CMP 纳米微球 ${ }^{[15]}$. 在较温和的细乳液反应条件下, 可以初步偶联聚合得到球形的超支化粒子，第二步将反 应溶液转移至水热釜中, 在较高温度与压力的水热条件 
下，促使支化粒子内未反应的单体或寡聚物进一步反应 交联, 得到官能团反应充分的 CMP 材料. 这种方法既 兼顾了在温和条件下形成粒径形态均一的微球, 又实现 了在剧烈反应条件下充分交联骨架, 相比之前仅通过乳 液聚合得到的 CMP 微球, 其多孔性得到了极大的提升. 在此基础上, 我们验证了该方法对于其他单体的适用 性. 四苯基乙烯分子在甲苯中原本溶解性一般, 很难聚 合得到多孔的 CMP 微球, 但是采用两步法进行水热处 理后, 得到的 CMP 微球比表面积在 $1200 \mathrm{~m}^{2} / \mathrm{g}$ 以上, 并 且聚集增强苂光的性质也得到增强.

韩国的 Seung Uk Son 课题组发展了模板法制备复 合结构的 CMP 微球. 不同于细乳液反应体系, 模板法 是通过沉淀聚合方式在模板粒子表面包覆一层 CMP 壳 层, 从而制备空心或是核壳结构的新型 CMP 材料. 他 们于 2013 年, 首次使用 $\mathrm{SiO}_{2}$ 微球作为模板, 在溶液中 通过 Sonogashira 偶联反应, 在模板表面沉淀聚合基于 四面体型的四苯基甲烷构筑的 $\mathrm{CMP}$, 再将内部 $\mathrm{SiO}_{2}$ 刻 蚀除去, 得到空心结构的 CMP 微胶囊(图 4) ${ }^{[16]}$.

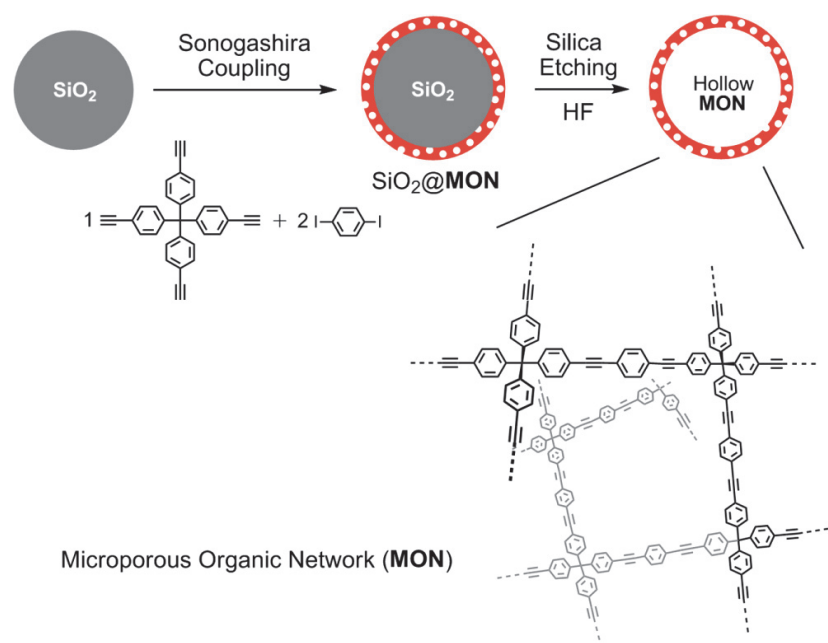

图 4 二氧化硅模板合成空心微孔有机骨架(改编自文献[16])

Figure 4 Synthetic route for the hollow microporous organic networks (H-MONs) using a silica template. (Adapted from the literature [16])

在 2014 年, 他们又使用 MOF 材料作为模板, 通过 表面包覆并刻蚀内部模板, 制备了方形的空心 CMP 材 料 ${ }^{[17]}$. 这种模板法一方面可以调控 CMP 微球的微观结 构, 另一方面可以将 CMP 作为模块, 基于工程模块组 装的思路将 CMP 优异的多孔性和光电性质引入复合物 中, 从而构筑新型的有机/无机杂化的功能复合微球.

总体而言, 借助于胶体化学已有的技术、研究储备, 将传统的合成方法推广到微纳尺度 CMP 的可控合成上, 是一条合理有效的研究思路; 而且除了可以调控微球的 形态、尺寸、结构以外, 还可以基于成熟的体系如乳液 聚合、沉淀聚合等, 尝试采用更多的聚合反应类型、聚 合单体、催化剂等合成多种的 CMP 纳米材料. 然而, 我 们也看到目前所研究的乳液体系, 常常使用较低极性的
溶剂作为油相(如甲苯), 由此对于单体的溶解以及聚合 反应程度都有较大的影响. 虽然通过水热条件处理可以 显著提高多孔性，然而该方法对于某些反应类型并没有 很好的效果. 同样，在模板上的沉淀聚合方法受到的限 制条件也较多, 其中反应溶剂、单体种类、模板表面理 化性质以及催化剂使用都会严重影响包覆效果. 因此, 发展微纳尺度 CMP 材料仍旧有大量工作需要探索和完 善.

\section{3 直接或间接方法制备 CMP 薄膜}

合成溶液可分散的纳米微球是解决 CMP 加工的一 个间接的方法, 目前仍需要与通用高分子或乳化剂共混 来制备涂层或薄膜材料，还没有得到基于 CMP 微球的 完全均相的 CMP 薄膜. 在这方面, 吉林大学朱广山教 授课题组将多孔芳香环骨架纳米粒子与易于成膜的聚 苯乙烯、聚甲基丙烯酸甲酯、聚乙烯基吡咯烷酮、聚醚 砜、聚氨酯、聚砜等高分子均匀混合，在基板上涂膜形 成兼具多孔性和机械强度的功能薄膜 ${ }^{[18]}$. 我们课题组 也将乳液聚合得到的 CMP 纳米粒子和聚乙烯醇水溶液 混合，得到可以发白光的 CMP 共混膜 ${ }^{[15]}$. 这种方法，虽 然 CMP 的自身性质会受到一定影响, 然而相比于直接 成膜的方法具有更大的灵活性.

在近几年中, 开发新的聚合方法、催化剂或是功能 单体使得 CMP 在合成中直接成膜是该领域研究的一个 热点. 2011 年, Park 课题组 ${ }^{[19]}$ 提出了一种设计巧妙的 bottom-up 制备方法. 利用两种四官能度的四面体型立 体小分子单体，基于一 $\mathrm{NH}_{2}$ 和一 $\mathrm{NCO}$ 这样的高活性反 应单元，用 layer-by-layer 的方式层层反应得到超薄聚合 物膜. 四面体的分子结构既带来了更加刚性的聚合物框 架结构，又从理论上确保反应基团两个朝向基板，两个 背离基板，从而实现层层反应，促使薄膜能够均匀生长， 最终得到 $36 \mathrm{~nm}$ 厚均匀的多孔薄膜. 基于这一方法原理, 在 2012 年, Kiriy 课题组 ${ }^{[20]}$ 用表面引发的方法合成了膜 厚为 $30 \mathrm{~nm}$ 左右的、完全共轭的 CMP 薄膜; 然而反应 类型和条件都有较多限制，较难推广到其他反应类型的 CMP 薄膜合成.

在 2012 年, 美国橡树岭国家实验室的戴胜教授课 题组使用新型的催化剂三氟甲磺酸催化氧基三聚交联 反应, 得到透明的三嗪连接的 CMP 薄膜, 低温氮气测 试表征其比表面积为 $738 \mathrm{~m}^{2} / \mathrm{g}$, 其中新型催化剂和低温 液相反应是成膜的关键条件 ${ }^{[21]}$, 此外也要求聚合单体 在反应温度下能够是液态. 因此, 对于单体的选择有较 多限制, 但作为制备 CMP 薄膜的代表性工作, 说明寻 找合适的催化体系也是一个重要思路.

华南理工大学马於光教授课题组 ${ }^{[22]}$ 采用了电化学 聚合的方法直接在基板上制备 CMP 薄膜. 他们成功通 过多次的伏安循环，氧化聚合由咔唑基团修饰的、四面 体型的四苯基甲烷分子, 得到了表面光滑、超薄、大面 积的 CMP 薄膜, 并且具有微孔通道及窄分布的孔径; 
通过电化学掺杂高氯酸根离子, 可以显著增加 CMP 薄 膜的导电率和功函数, 从而作为电极中间层, 可以提高 聚合物光伏电池以及发光二极管的效率. 但其局限性在 于适用的单体较少, 目前报道的体系都只限于使用带有 咔唑基团的单体进行聚合反应，分子设计方面受到一定 限制.

日本分子科学研究所的江东林教授课题组 ${ }^{[23]}$ 同样 采用电化学氧化聚合咔唑修饰的三苯基苯, 得到透明柔 性的、自支撑 CMP 发光薄膜. 这种薄膜可以通过增加 伏安循环的次数来精准调控膜的厚度, 每次循环可增厚 $2.5 \mathrm{~nm}$, 当厚度超过 $50 \mathrm{~nm}$ 时, 薄膜就可从基板上揭下 成为自支撑膜(图 5). 这样制膜的方法非常实用，且作为 一种多孔材料, 其用氪气吸附表征得到的比表面积高达 $1450 \mathrm{~m}^{2} / \mathrm{g}$. 这种发蓝色荧光的多孔薄膜还可以用于光 学传感，显示出对芳香化合物、金属离子、多巴胺、次 氯酸等快速、灵敏、稳定的苂光性质变化.
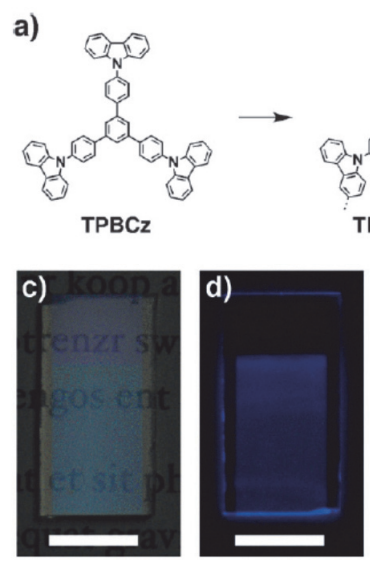
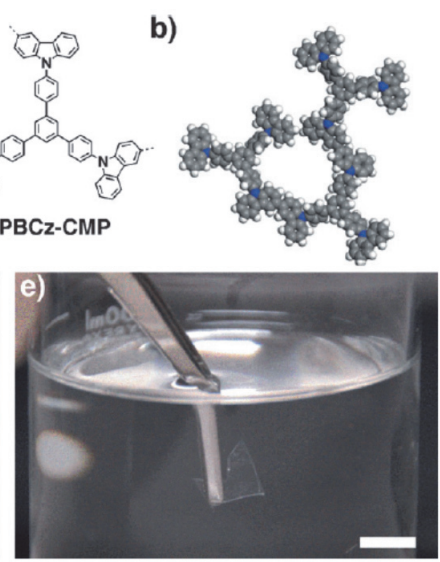

图 5 (a) TPBCz-CMP 薄膜的合成示意图; (b)薄膜内孔的基本单元模 拟图; (c)在 ITO 基板上的薄膜照片; (d)紫外灯下的薄膜照片; (e)能够 自支撑的 $100 \mathrm{~nm}$ 薄膜照片(改编自文献[23], 标尺 $0.5 \mathrm{~cm}$ )

Figure 5 (a) Schematic representation of the electrochemical synthesis of TPBCz-CMP films. (b) Elementary pore of the film. Photos of (c) a film on an ITO substrate, (d) the film under UV light, and (e) a $100 \mathrm{~nm}$ thick free-standing film in acetonitrile. (Adapted from the literature [23]) Scale bars: $0.5 \mathrm{~cm}$.

总体而言，由于纳米尺度 CMP 合成技术逐渐成熟， 将其分散液与通用高分子共混制备 CMP 复合膜的方法 就变得更加简便有效; 不仅可以通过各种传统工艺进行 加工处理, 而且保持了一定的机械强度以及不同 CMP 带来的多样化功能, 因此将会基于复合薄膜或涂层的形 式来展现 CMP 的实用价值和优异性能. 相比而言, 在 CMP 聚合过程中直接制膜的研究才刚刚起步. 可以看 出, 特定条件控制的溶液聚合、电化学聚合、或是表面 引发等新方法, 都对单体或聚合体系有特殊的要求, 同 时反应类型也有较大局限. 不过, 相比于复合膜, 这种 完全由 CMP 骨架构筑的薄膜由全共轭高分子支撑, 因 此在组装成器件后将会显示出优异的光电相关的性能.

\section{4 有机相 CMP 化学凝胶的合成}

将 CMP 制备成凝胶是提高其溶液性质，并实现 CMP 加工成型的有效策略. 我们课题组首次报道了通 过炔炔氧化偶联反应聚合四炔基四苯基锌卟啉单体，制 备得到了刚性骨架组成的弹性有机化学凝胶 ${ }^{[24]}$. 去除 溶剂后的干凝胶在微观结构上是由纤维相互编织而成 的凝胶网络，但在分子水平上完全与此前报道的 CMP 粉末材料相同，具有三维交联的高分子骨架和稳定的多 孔特征. 并且该方法具有一定的普适性，使用简单的均 三炔基苯分子也可以聚合得到相应的化学凝胶. 在此基 础上, 可以将锌卟啉和均三炔基苯两组分单体混合, 在 室温下催化聚合, 先得到锌卟啉的凝胶, 然后升高温度 使得均三炔基苯在凝胶中聚合，从而获得两种 CMP 的 互穿网络结构(图 6). 除了四氢呋喃，丙酮、二甲基甲酰 胺、二甲基亚砜、二氧六环、甲苯等多种常见溶剂也可 用于化学凝胶的反应，并且温度和单体浓度的改变可以 有效调节干凝胶的多孔性. 我们认为这种方法还可以进 一步扩大单体和反应类型，合成更多种类的 CMP 凝胶 材料. 而且, 基于 CMP 凝胶的合成策略还具有以下三 个方面的优势，一是凝胶过程中，可以在液相中复合如 碳纳米管、石墨烯等多维尺度的高性能碳材料，从而丰 富 CMP 的功能化; 二是，除了平面基板，也可以在三维 网络中进行凝胶聚合，如多孔海绵、泡沫镍、碳衣等复 合用于器件的制备; 三是，在 CMP 凝胶中进行孔道的 功能化修饰要比 CMP 固体粉末具有更高的效率和功能 基团密度.

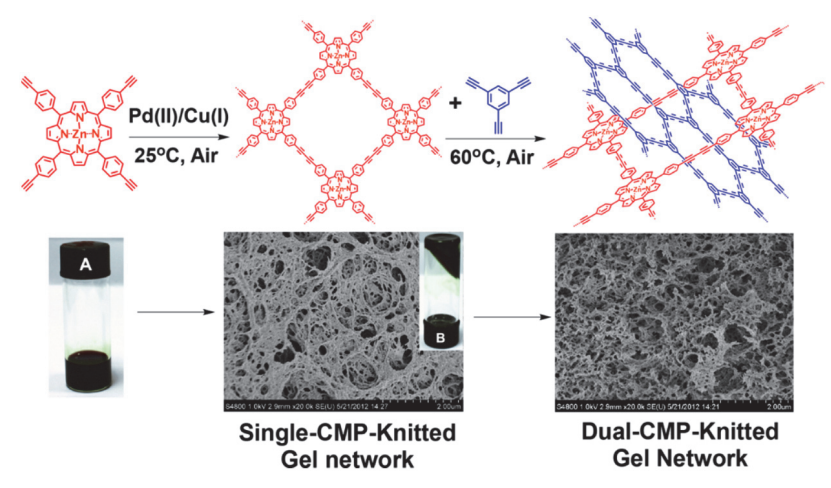

图 6 四炔基四苯基锌卟啉单体合成 CMP 凝胶网络以及该单体与均 三炔基苯单体形成互穿网络凝胶的示意图(改编自文献[24])

Figure 6 Schematic representation of the electrochemical synthesis of ZnPor-CMP gel network and Dual-CMP interpenetrating network gel. (Adapted from the literature [24])

\section{3 总结与展望}

CMP 材料作为一类无定型结构的新型有机多孔材 料, 科研工作者不断从聚合单体、反应类型、聚合方法、 催化剂以及功能化等方面着手进行了大量的探索，同时 对其溶液性质和加工问题展开了不断的尝试. 近几年 
间, 共有四种不同的思路可控合成 CMP 来解决这一难 题.

可溶性 CMP 是较为理想的材料加工形式, 但对于 目前所报道的可溶性 CMP 来说, 更近似于超支化聚合 物的结构, 因此后处理的过程会对刚性骨架堆叠的微孔 结构有较大影响. 在引入先预聚加工成膜, 再交联成网 络结构的方法之后, 对于制备平整且内部具备多孔性的 交联薄膜非常具有实际意义.

微纳尺度 CMP 微球的合成可采用乳液聚合、沉淀 聚合等成熟的胶体制备技术，可适用于较多的单体以及 反应类型, 产物体现了高比表面积、微孔特征、可调控 的微球结构以及共轭高分子的特性, 不仅具有很好的溶 液分散性和可修饰性, 能与通用高分子共混制备复合涂 层或是薄膜, 而且在多个领域都展现了微纳尺度 CMP 在液相体系中独特而优异的实用价值. 目前, 如何进一 步提高 CMP 纳米材料的种类和多孔性、如何基于模块 组装思路丰富 CMP 复合纳米材料的功能性, 是发展新 型微观尺度 CMP 材料的重要科学问题.

相比于 CMP 复合膜的研究工作, 在聚合中使 CMP 在基板上直接成膜, 会更加吸引研究工作者的兴趣. 目 前, 最成功的例子是采用电化学聚合的方法制备 CMP 薄膜, 已成功用于器件的制备, 并显著提高了光电转换 效率和传感功能. 该方法只适合电化学活性的单体, 因 此对于报道过的大部分 CMP 体系并不适用, 但如果专 注于某些特定的应用场合, 该方法非常实用. 此外, 新 型催化剂以及聚合方法的开发, 也将是直接合成 CMP 薄膜的重要挑战和主要发展思路.

CMP 的凝胶形式不仅具有内在的共轭网络骨架, 并被充分溶剂化, 展示了典型的化学凝胶的特点; 同时 在制备 CMP 凝胶的过程中, 可以更加灵活、方便、高 效的复合功能材料, 或是实现在特殊基板上的原位凝胶 成型; 此外, 溶剂化的 CMP 骨架也容易实现孔道内的 功能化修饰. 因此, 相比于传统的 CMP 材料, 通过炔基 偶合制备凝胶 CMP, 在后续的应用中会有更好的发展 前景; 但是相关的研究才刚刚起步, 目前还缺乏实用方 面的大量探索和尝试.

新材料的发展是推动社会进步的强大动力, 一种新 材料很有可能从根本上改变我们的生活方式. CMP 材料 问世的七年以来, 最初只是作为气体或化学物质的吸附 分离材料, 然而随着 CMP 溶液性质的提高以及不同形 式 CMP 的合成, 科研工作者可以对其进行加工、组装、 复合, 使其有希望进入更广泛的应用领域. 这其中, 许 多国内的科研工作者提出了很多解决问题的思路, 并做 出了重要的引领前沿的工作. 从目前的研究进展来看, CMP 材料非常有希望成为真正有实用价值的功能性聚 合物材料. 在此, 我们抛砖引玉, 介绍以上的最新进展, 希望阅读本文的学者们能够在此基础上更进一步, 研究 出更多的解决方案来有效使用 CMP 材料.
作者简介

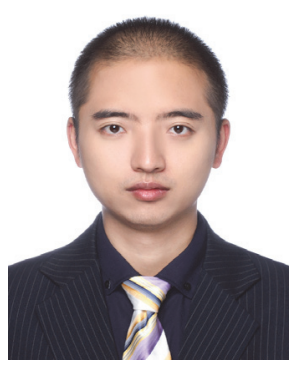

吴可义, 1983 年, 复旦大学高分子科学系博士生. 2006 年 本科毕业于复旦大学高分子材料与工程专业, 2011 年开始在 复旦大学高分子科学系攻读硕士学位, 2013 年转为博士生, 攻 读高分子化学与物理专业博士学位. 研究方向为有机多孔聚 合物材料.

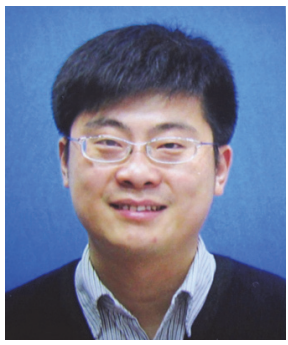

郭佳, 复旦大学高分子科学系副教授. 2002 年毕业于上海 大学获高分子材料与工程学士学位, 2007 年毕业于复旦大学 获得高分子化学与物理专业博士学位; 同年得到日本学术振 兴会(JSPS)外国人特别研究员项目资助，于 2007-2009 年期 间在日本国立自然科学机构分子科学研究所从事博士后研究 工作, 导师江东林教授; 2009 年 10 月加入复旦大学高分子科 学系工作. 其研究兴趣是发展多种形式的多孔有机材料以及 有机/无机杂化的功能性聚合物复合微球.

\section{References}

[1] (a) Li, H.; Eddaoudi, M.; Groy, T. L.; Yaghi, O. M. J. Am. Chem. Soc. 1998, 120, 8571; (b) Liu, B.; Tang, L. X.; Lian, Y. H.; Li, Z.; Sun, C. Y.; Chen, G. J. Acta Chim. Sinica 2013, 71, 920 (刘蓓, 唐李 兴, 廉源会, 李智, 孙长宇, 陈光进, 化学学报, 2013, 71, 920); (c) Jia, J. T.; Wang, L.; Zhao, Q.; Sun, F. X.; Zhu, G. S. Acta Chim. Sinica 2013, 71, 1492 (贾江涛, 王蕾, 赵晴, 孙福兴, 朱广山, 化 学学报, 2013, 71, 1492); (d) Liu, B.; Lian, Y. H.; Li, Z.; Chen, G. J. Acta Chim. Sinica 2014, 72, 942 (刘蓓, 廉源会, 李智, 陈光进, 化 学学报, 2014, 72, 942); (e) He, Y. P.; Tan, Y. X.; Zhang, J. Acta Chim. Sinica 2014, 72, 1228 (何燕萍, 谭衍曦, 张健, 化学学报, 2014, 72, 1228).

[2] (a) Thomas, A. Angew. Chem., Int. Ed. 2010, 49, 8328; (b) Dawson, R.; Cooper, A. I.; Adams, D. J. Prog. Polym. Sci. 2012, 37, 530; (c) Zhang, T. T.; Wang, H. T.; Ma, H. P.; Sun, F. X.; Cui, X. Q.; Zhu, G. S. Acta Chim. Sinica 2013, 71, 1598 (张婷婷, 王海涛, 马和平, 孙 福兴, 崔小强, 朱广山, 化学学报, 2013, 71, 1598); (d) Wang, W.; Yan, Z. J.; Yuan, Y.; Sun, F. X.; Zhao, M.; Ren, H.; Zhu, G. S. Acta Chim. Sinica 2014, 72, 557 (王维, 间卓君, 元野, 孙福兴, 赵明, 任浩, 朱广山, 化学学报, 2014, 72, 557.).

[3] (a) Cote, A. P.; Benin, A. I.; Ockwig, N. W.; O'keeffe, M.; Matzger, A. J.; Yaghi, O. M. Science 2005, 310, 1166; (b) El-Kaderi, H. M.; Hunt, J. R.; Mendoza-Cortes, J. L.; Cote, A. P.; Taylor, R. E.; O'keeffe, M.; Yaghi, O. M. Science 2007, 316, 268; (c) Wan, S.; Guo, J.; Kim, J.; Ihee, H.; Jiang, D. Angew. Chem. Int. Ed. 2008, 47, 8826; (d) Wan, S.; Guo, J.; Kim, J.; Ihee, H.; Jiang, D. Angew. Chem., Int. Ed. 2009, 48, 5439; (e) Uribe-Romo, F. J.; Doonan, C. J.; Furukawa, H.; Oisaki, K.; Yaghi, O. M. J. Am. Chem. Soc. 2011, 
133, 11478; (f) Ding, S.; Gao, J.; Wang, Q.; Zhang, Y.; Song, W.; Su, C.; Wang, W. J. Am. Chem. Soc. 2011, 133, 19816; (g) Ding, X.; Guo, J.; Feng, X.; Honsho, Y.; Guo, J.; Seki, S.; Maitarad, P.; Saeki, A.; Nagase, S.; Jiang, D. Angew. Chem., Int. Ed. 2011, 50, 1289; (h) Colson, J. W.; Woll, A. R.; Mukherjee, A.; Levendorf, M. P.; Spitler, E. L.; Shields, V. B.; Spencer, M. G.; Park, J.; Dichtel, W. R. Science 2011, 332, 228; (i) Spitler, E. L.; Colson, J. W.; Uribe-Romo, F. J.; Woll, A. R.; Giovino, M. R.; Saldivar, A.; Dichtel, W. R. Angew. Chem., Int. Ed. 2012, 51, 2623; (j) Feng, X.; Ding, X.; Jiang, D. Chem. Soc. Rev. 2012, 41, 6010; (k) Guo, J.; Xu, Y.; Jin, S.; Chen, L.; Kaji, T.; Honsho, Y.; Addicoat, M. A.; Kim, J.; Saeki, A.; Ihee, H.; Seki, S.; Irle, S.; Hiramoto, M.; Gao, J.; Jiang, D. Nat. Commun. 2013, 4, 2736; (1) Ding, S.; Wang, W. Chem. Soc. Rev. 2013, 42, 548.

[4] (a) Tsyurupa, M. P.; Davankov, V. A. React. Funct. Polym. 2002, 53, 193; (b) Germain, J.; Frechet, J. M. J.; Svec, F. J. Mater. Chem. 2007, 17, 4989; (c) Lee, J. Y.; Wood, C. D.; Bradshaw, D.; Rosseinsky, M. J.; Cooper, A. I. Chem. Commun. 2006, 2670; (d) Fontanals, N.; Manesiotis, P.; Sherrington, D. C.; Cormack, P. G. Adv. Mater. 2008, 20, 1298; (e) Germain, J.; Svec, F.; Frechet, J. M. J. Chem. Mater. 2008, 20, 7069.

[5] (a) Mckeown, N. B.; Makhseed, S.; Budd, P. M. Chem. Commun. 2002, 2780; (b) Mckeown, N. B.; Hanif, S.; Msayib, K.; Tattershall, C. E.; Budd, P. M. Chem. Commun. 2002, 2782; (c) Budd, P. M.; Ghanem, B. S.; Makhseed, S.; Mckeown, N. B.; Msayib, K. J.; Tattershall, C. E. Chem. Commun. 2004, 230; (d) Budd, P. M.; Ghanem, B.; Msayib, K.; Mckeown, N. B.; Tattershall, C. J. Mater. Chem. 2003, 13, 2721; (e) Ghanem, B. S.; Msayib, K. J.; Mckeown, N. B.; Harris, K. D. M.; Pan, Z.; Budd, P. M.; Butler, A.; Selbie, J.; Book, D.; Walton, A. Chem. Commun. 2007, 67; (f) Mckeown, N. B.; Gahnem, B.; Msayib, K. J.; Budd, P. M.; Tattershall, C. E.; Mahmood, K.; Tan, S.; Book, D.; Langmi, H. W.; Walton, A. Angew. Chem. Int. Ed. 2006, 45, 1804; (g) Ghanem, B. S.; Hashem, M.; Harris, K. D. M.; Msayib, K. J.; Xu, M.; Budd, P. M.; Chaukura, N.; Book, D.; Tedds, S.; Walton, A.; Mckeown, N. B. Macromolecules 2010, 43, 5287; (h) Ghanem, B. S.; Mckeown, N. B.; Budd, P. M.; Fritsch, D. Macromolecules 2008, 1640; (i) Carta, M.; Msayib, K. J.; Budd, P. M.; Mckeown, N. B. Org. Lett. 2008, 10, 2641; (j) Ghanem, B. S.; Mckeown, N. B.; Budd, P. M.; Al-Harbi, N. M.; Fritsch, D.; Heinrich, K.; Starannikova, L.; Tokarev, A.; Yampolskii, Y. Macromolecules 2009, 42, 7881; (k) Weber, J.; Su, O.; Antonietti, M.; Thomas, A. Macromol. Rapid Commun. 2007, 28, 1871; (1) Ritter, N.; Senkovska, I.; Kaskel, S.; Weber, J. Macromol. Rapid Commun. 2011, 32, 438.

[6] (a) Jiang, J.; Su, F.; Trewin, A.; Wood, C. D.; Campbell, N. L.; Niu, H.; Dickinson, C.; Ganin, A. Y.; Rosseinsky, M. J.; Khimyak, Y. Z.; Cooper, A. I. Angew. Chem. Int. Ed. 2007, 46, 8574; (b) Jiang, J.; Su, F.; Trewin, A.; Wood, C. D.; Niu, H.; Jones, J. T. A.; Khimyak, Y. Z.; Cooper, A. I. J. Am. Chem. Soc. 2008, 130, 7710; (c) Weder, C. Angew. Chem., Int. Ed. 2008, 47, 448; (d) Weber, J.; Thomas, A. J. Am. Chem. Soc. 2008, 130, 6334; (e) Schmidt, J.; Werner, M.; Thomas, A. Macromolecules 2009, 42, 4426; (f) Schmidt, J.; Weber, J.; Epping, J. D.; Antonietti, M.; Thomas, A. Adv. Mater. 2009, 21, 702; (g) Cooper, A. I. Adv. Mater. 2009, 21, 1291; (h) Stoeckel, E.; Wu, X.; Trewin, A.; Wood, C. D.; Clowes, R.; Campbell, N. L.; Jones, J. T. A.; Khimyak, Y. Z.; Adams, D. J.; Cooper, A. I. Chem. Commun. 2009, 212; (i) Ben, T.; Ren, H.; Ma, S.; Cao, D.; Lan, J.; Jing, X.; Wang, W.; Xu, J.; Deng, F.; Simmons, J. M.; Qiu, S.; Zhu, G. Angew. Chem., Int. Ed. 2009, 48, 9457; (j) Holst, J. R.; Stoeckel, E.; Adams, D. J.; Cooper, A. I. Macromolecules 2010, 43, 8531; (k) Chen, L.; Honsho, Y.; Seki, S.; Jiang, D. J. Am. Chem. Soc. 2010, 132, 6742; (1) Yuan, D.; Lu, W.; Zhao, D.; Zhou, H. Adv. Mater. 2011, 23, 3723; (m) Cheng, G.; Hasell, T.; Trewin, A.; Adams, D. J.; Cooper, A. I. Angew. Chem., Int. Ed. 2012, 51, 12727; (n) Kiskan, B.; Weber, J. ACS Macro Lett. 2012, 1, 37; (o)Xu, Y.; Jin, S.; Xu, H.; Nagai, A.; Jiang, D. Chem. Soc. Rev. 2013, 42, 8012; (p) Gu, C.; Huang, N.; Gao, J.; Xu, F.; Xu, Y.; Jiang, D. Angew. Chem., Int. Ed. 2014, 53, 4850.

[7] (a) Jiang, J.; Su, F.; Niu, H.; Wood, C. D.; Campbell, N. L.; Khim- yak, Y. Z.; Cooper, A. I. Chem. Commun. 2008, 486; (b) Yuan, S.; Dorney, B.; White, D.; Kirklin, S.; Zapol, P.; Yu, L.; Liu, D. Chem. Commun. 2010, 46, 4547; (c) Hasell, T.; Wood, C.; Clowes, R.; Jones, J.; Khimyak, Y.; Adams, D.; Cooper, A. I. Chem. Mater. 2010, 22, 557; (d) Li, A.; Wang, L.; Wang, X.; Han, K.; Deng, W. Angew. Chem. Int. Ed. 2010, 49, 3330; (e) Chen, Q.; Luo, M.; Wang, T.; Wang, J.; Zhou, D.; Han, Y.; Zhang, C.; Yan, C.; Han, B. Macromolecules 2011, 44, 5573; (f) Chen, Q.; Luo, M.; Hammershøj, P.; Zhou, P.; Han, Y.; Laursen, B. W.; Yan, C.; Han, B. J. Am. Chem. Soc. 2012, 134, 6084; (g) Liu, X.; Xu, Y.; Guo, Z.; Nagai, A.; Jiang, D. Chem. Commun. 2013, 49, 3233; (h) Xiang, Z.; Cao, D. J. Mater. Chem. A 2013, 1, 2691.

[8] (a) Palkovits, R.; Antonietti, M.; Kuhn, P.; Thomas, A.; Schüth, F. Angew. Chem. Int. Ed. 2009, 48, 6909; (b) Chen, L.; Yang, Y.; Jiang, D. J. Am. Chem. Soc. 2010, 132, 9138; (c) Du, X.; Sun, Y.; Tan, B.; Teng, Q.; Yao, X.; Su, C.; Wang, W. Chem. Commun. 2010, 46, 970; (d) Chen, L.; Yang, Y.; Guo, Z.; Jiang, D. Adv. Mater. 2011, 23, 3149; (e) Jiang, J.; Wang, C.; Laybourn, A.; Hasell, T.; Clowes, B.; Cooper, A. I. Angew. Chem. Int. Ed. 2011, 50, 1072; (f) Zhang, P.; Weng, Z.; Guo, J.; Wang, C. Chem. Mater. 2011, 23, 5243; (g) Zhang, Y.; Zhang, Y.; Sun, Y.; Du, X.; Shi, J.; Wang, W.; Wang, W. Chem. Eur. J. 2012, 18, 6328; (h) Zhang, K.; Kopetzki, D.; Seeberger, P.; Antonietti, M.; Vilela, F. Angew. Chem., Int. Ed. 2013, 52, 1432; (i) Urakami, H.; Zhang, K.; Vilela, F. Chem. Commun. 2013, 49, 2353.

[9] (a) Xu, Y.; Chen, L.; Cuo, Z.; Nagai, A.; Jiang, D. J. Am. Chem. Soc. 2011, 133, 17622; (b) Jiang, J.; Trewin, A.; Adams, D.; Cooper, A. I. Chem. Sci. 2011, 2, 1777; (c) Chen, Q.; Wang, J.; Yang, F.; Zhou, D.; Bian, N.; Zhang, X.; Yan, C.; Han, B. J. Mater. Chem. 2011, 21, 13554; (d) Zhang, K.; Tieke, B.; Vilela, F.; Skabara, P. Macromol. Rapid Commun. 2011, 32, 825; (e) Xu, Y.; Nagai, A.; Jiang, D. Chem. Commun. 2013, 49, 1591.

[10] (a) Feng, X.; Liang, Y.; Zhi, L.; Thomas, A.; Wu, D.; Lieberwirth, I.; Kolb, U.; Müllen, K. Adv. Funct. Mater. 2009, 19, 2125; (b) Kou, Y.; Xu, Y.; Guo, Z.; Jiang, D. Angew. Chem. Int. Ed. 2011, 50, 8753.

[11] (a) Liu, X.; Xu, Y.; Jiang, D. J. Am. Chem. Soc. 2012, 134, 8738; (b) Xiang, Z.; Cao, D. Macromol. Rapid Commun. 2012, 33, 1184; (c) Zhang, P.; Guo, J.; Wang, C. J. Mater. Chem. 2012, 22, 21426; (d) Wu, K.; Guo, J.; Wang, C. Chem. Commun. 2014, 50, 695.

[12] Figueira-Duarte, T. M.; Simon, S. C.; Wagner, M.; Drtezhinin, S. I.; Zachariasse, K. A.; Muellen, K. Angew. Chem., Int. Ed. 2008, 47, 10175.

[13] Cheng, G.; Bonillo, B.; Sprick, R. S.; Adams, D. J.; Hasell, T.; Cooper, A. I. Adv. Funct. Mater. 2014, 24, 5219.

[14] Patel, H. A.; Ko, D.; Yavuz, C. T. Chem. Mater. 2014, 26, 6729.

[15] Zhang, P.; Wu, K.; Guo, J.; Wang, C. ACS Macro Lett. 2014, 3, 1139.

[16] Kang, N.; Park, J.; Jin, M.; Park, N.; Lee, S.; Kim, H.; Kim, J.; Son, S. J. Am. Chem. Soc. 2013, 135, 19115.

[17] Chun, J.; Kang, S.; Park, N.; Park, E.; Jin, X.; Kim, K.; Seo, H.; Lee, S.; Kim, H.; Kwon, W.; Park, Y.; Kim, J.; Kim, Y.; Son, S. J. Am. Chem. Soc. 2014, 136, 6786.

[18] (a) Yuan, Y.; Sun, F.; Zhang, F.; Ren, H.; Guo, M.; Cai, K.; Jing, X.; Gao, X.; Zhu, G. Adv. Mater. 2013, 25, 6619; (b) Gao, X.; Zou, X.; Ma, H.; Meng, S.; Zhu, G. Adv. Mater. 2014, 26, 3644.

[19] Kim, M.; Byeon, M.; Bae, J.; Moon, S.; Yu, G.; Shin, K.; Basarir, F.; Yoon, T.; Park, J. Macromolecules 2011, 44, 7092.

[20] Senkovskyy, V.; Senkovska, I.; Kiriy, A. ACS Macro Lett. 2012, 1, 494.

[21] Zhu, X.; Tian, C.; Mahurin, S. M.; Chai, S.; Wang, C.; Brown, S.; Veith, G. M.; Luo, H.; Liu, H.; Dai, S. J. Am. Chem. Soc. 2012, 134, 10478 .

[22] Gu, C.; Chen, Y.; Zhang, Z.; Xue, S.; Sun, S.; Zhang, K.; Zhong, C.; Zhang, H.; Pan, Y.; Lv, Y.; Yang, Y.; Li, F.; Zhang, S.; Huang, F.; Ma, Y. Adv. Mater. 2013, 25, 3443.

[23] Gu, C.; Huang, N.; Gao, J.; Xu, F.; Xu, Y.; Jiang, D. Angew. Chem., Int. Ed. 2014, 53, 4850.

[24] Wu, K.; Guo, J.; Wang, C. Chem. Mater. 2014, 26, 6241. 